\title{
VLBI OBSERVATIONS OF RADIO STARS
}

\author{
Jean-François Lestrade \\ Bureau des Longitudes \\ 77 Avenue Denfert-Rochereau, F-75014, Paris, France \\ and Jet Propulsion Laboratory, Pasadena, CA
}

\section{INTRODUCTION}

In recent years, observations of stars across the whole electromagnetic spectrum have yielded a wealth of information on their atmospheres and borne out new insights on their internal structures. Indirect evidences for chromospheres, coronae and winds were discovered in stars other than the Sun. For instance, ultra-violet and optical emission lines from collisionally excited atoms are evidences for chromospheric activity with temperatures between $10^{4}$ and $2 \times 10^{5} \mathrm{~K}$. Soft X-ray emission with thermal spectrum is evidence for hot coronae with temperatures between $10^{6}$ and $3 \times 10^{7} \mathrm{~K}$. The spindown of stars with age is best interprated by loss of mass and of angular momentum via magneticly coupled stellar winds originating in hot stellar coronae.

It is common practice to divide the stars into early-type (earlier than spectral type F0) and late-type. The early-type stars have massive winds driven by their intense photospheric radiative flux. The discovery of X-ray emitting gas from these stars was a surprise. It is currently interprated as hot gas confined by small scale magnetic loops while the radiatively driven wind escapes through the "holes" between them. The late-type stars have atmospheres which are dominated by chromospheric and coronal activities and have relatively moderate winds. It is thought that magnetic fields shape the atmospheres and control the energetics of late-type stars. The corona is considered an assemblage of magnetic loops emerging from the stellar surface and confining a plasma which is too hot to be gravitationally bound to the star. The magnetic fields at the stellar surface are stressed by turbulent fluid motions and generate heating of the chromosphere and corona by dissipation of DC current or/and MHD waves. The moderate wind escapes through coronal holes which are regions of open magnetic lines between the loops.

At radio wavelengths, stellar thermal emission has been detected from early-type stars and is an important tool to determine their mass loss rates. The large scale winds and ionized circumstellar envelopes of these stars are best studied at the Very Large Array with arcsecond resolution. We shall not review these observations and refer the reader to Abbott (1985) and Kwok (1985). Stellar non-thermal continuum radio emission has been detected from single late-type stars and from close binaries having at least a late-type component. This non-thermal radio activity is sporadic and occurs on the Sun too where it is interprated with a variety of coherent and incoherent gyromagnetic emission mechanisms (Dulk 1985). We shall review the contributions of VLBI to a better understanding of the emission process(es) and of the physical properties of the non-thermal radio stars. We exclude pulsars, stellar molecular masers and super nova remnants from this discussion.

The RS CVn (RS Canum Venaticorum) close binaries are the most active non-thermal 
emitters and most VLBI observations of radio stars have been conducted on them. They are detectable at distances of less than 100 parsecs and their angular sizes are between 1 and 3 milliarcseconds matching the angular resolution of VLBI. The relatively successful VLBI observations of these close binaries in recent years have come with the implementation of the Mark III VLBI data acquisition system (Rogers et al 1983). This system provides the sensitivity required for these objects which are weak by VLBI standards (typically $<100 \mathrm{mJy}$ ).

VLBI observations of RS CVn are motivated both by astrophysic and astrometric goals. In astrophysics, direct measurements by VLBI of their radio source sizes and of their brightness temperatures as well as imaging of their milliarcsecond radio emitting regions are helping to understand their emission mechanism which produces at least $10^{4}$ times more energy than the Sun at radio wavelengths. VLBI observations yield also the values of important model-dependent parameters, e.g. magnetic field strength and electron number density, and will possibly unveil the nature of the magnetic structure which might be the dominant energy source in late-type stars. In astrometry, the RS CVn binaries being both radio active and optically bright can be used as link objects to tie the radio and optical celestial reference frames together. The quality of such a tie depends on the degree of coincidence between the optical and radio components. This can be answered with a comprehensive physical model of these stars.

Five X-ray stars (Cyg X3, Cyg X1, Circ X1, LSI $61^{\circ} 303$ and Sco X1) and the exotic objects SS 433 have also been studied by VLBI technique. These objects have uncommon properties and cannot be easily classified. Their energetics might be analogous, although on a different scale, to the mechanisms powering the extragalactic sources. Hence, they are an interesting connection with the physics of extragalatic radio sources.

\section{RS CVn AND ALGOL CLOSE BINARIES}

\section{a. PHYSICAL PROPERTIES}

RS CVn close binaries (Fig. 1a) have detached stellar components with mass ratio close to unity. Both components are late-type stars, typically GV for the primary and KOIV for the secondary, tidally locked with rotation-orbital periods generally less than 20 days. These close binaries were first noticed for the conspicuous wavelike distorsions of their light curves (Hall 1975) presumably caused by large dark starspots on the photosphere of the K-subgiant. The evolution of these starspots (in size and location) has been studied by photometry (Rodonò 1983) and recently by a Doppler imaging technique (Vogt and Penrod 1983). Algol (Fig. 1b) is as active in radio as RS CVn but is a semi-detached close binary (belonging to a multiple system) characterized by a late-type component filling its Roche lobe and spilling over gas on an early-type star through an accretion disc.

Radio surveys of RS CVn binaries were undertaken by Hjellming, Gibson, Spangler, Owens and Feldman with single dishes or short baseline interferometers in the 70's. Nonthermal outbursts with flux density ranging from a few tens to a few hundreds mJy, and exceptionally up to $1.2 \mathrm{Jy}$ for HR1099, were discovered by these astronomers. The corresponding radio luminosity is between $2 \times 10^{15}$ and $6 \times 10^{17} \mathrm{ergs} / \mathrm{s} / \mathrm{Hz}$ or $10^{4}$ to $10^{6}$ times larger than the Sun. A dozen of radio RS CVn were discovered during these early surveys. Later, the sensitivity of the VLA and its imaging capability to eliminate confusion sources led to the discovery of more than 30 active RS CVn compiled in Mutel and Lestrade (1985).

Pallavicini (1986) in his review of coronal magnetic fields stresses that early-type stars have no convective zone while late-type stars do have such a zone. This leads to think that the dominant energy source in late-type stars, as RS CVn and Algol-type, might be 
a dynamo mechanism involving the interaction of rotation and convection. Such interaction generates intense magnetic fields emerging at the stellar surface and penetrating through all layers of the stellar atmosphere. Stellar components of close binary have much higher rotation rates than single star with the same spectral type because rotation is maintained against spindown by tidal torque. This rapid rotation increases the efficiency of the dynamo mechanism and might be responsible for the exceptional magnetic activity of RS CVn as evidenced by their starspots which might cover up to $30 \%$ of their surface and should be associated with intense magnetic fields. The variable degree of circular polarization observed from RS CVn, usually between 0 and $30 \%$ and exceptionally more, is another evidence for large magnetic structure. Mutel et al (1987) have noticed than the sense of circular polarization at a given frequency stays the same over several years. This is evidence for the existence of permanent large magnetic structures on these stars.

The very hot $\mathrm{X}$-ray coronae $\left(\leq 3 \times 10^{7} \mathrm{~K}\right)$ discovered in RS CVn have been estimated to be at least 3 stellar radii in extent for the $\mathrm{RS} C \mathrm{CV}$ system AR Lac (Walter, Gibson and Basri 1983) and for Algol (White et al 1985) when X-

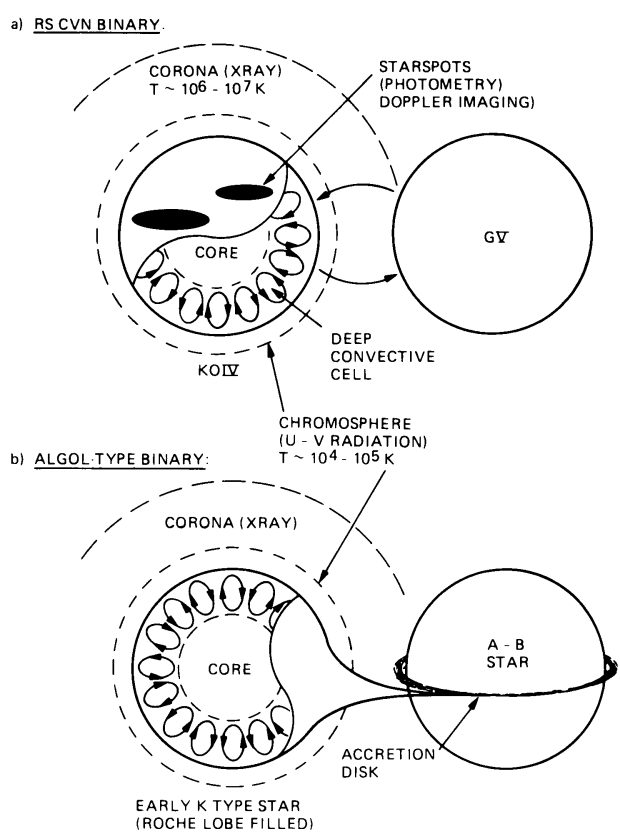

Fig. 1: sketches of RS CVn and Algo1-type close binary systems. ray eclipses failed to be detected during optical eclipses of these systems. This is unlike the solar magnetic structures which have scale heights much smaller than a solar radius $\left(<0.01 R_{\odot}\right)$. Such linear sizes correspond to milliarcsecond extent at the distances of these RS CVn. The lack of spatial resolution of X-ray observations makes the topology of coronal magnetic fields confining hot plasma unknown in stars other than the Sun. VLBI observations can acheive milliarcsecond resolution in radio and therefore is presently the only technique capable of probing the magnetic structures of these non-thermal stars.

\section{b. VLBI OBSERVATIONS OF CLOSE BINARIES}

The first radio star observed by the VLBI technique was Algol by Clark B.G., Kellermann and Shaffer (1975) and Clark T.A. et al (1976) at $8.4 \mathrm{GHz}$ on a single baseline during two exceptional outbursts $(>0.5 \mathrm{Jy}$ ). The fringe visibility functions they obtained provided the first direct measurements of the radio source size (5 mas and 2 mas) and of the brightness temperature $\left(T_{B}>10^{8} \mathrm{~K}\right)$ for continuum radio emission from a star. The important result was that $T_{B}$ was high enough to be clear evidence for a non-thermal emission mechanism. The data were also used to derive the VLBI position of Algol (Clark et al 1976) at epoch B1950 and were recently re-analysed by Ma et al (1986) in the J2000 
frame.

In 1978, Mark II VLBI observations were successfully conducted on the two most active RS CVn; UX Ari by Backer and Srameck at $1.4 \mathrm{GHz}$ between Arecibo and Greenbank (unpublished) and HR1099 by Resch at $8.4 \mathrm{GHz}$ between Goldstone and Owens Valley (unpublished). The visibility functions were thought unreliable by these authors because of lack of proper flux scale calibration and so neither source sizes nor brightness temperatures were inferred from these data. In 1981, Cohen detected the RS CVn system HR5110 during Mark III VLBI observations at $5 \mathrm{GHz}$ between Owens Valley and Greenbank and inferred a source size of 3.7 milliarcseconds (Little-Marenin et al 1986).

In December 1982, we initiated a series of VLBI observations with the Mark III recording system and the US VLB Network including stations in Europe and from the Deep Space Network. These VLBI observations were conducted at various centimetric wavelengths and sometimes at dual frequency and dual polarization. The first five experiments were designed to survey about twenty RS CVn and Algol-type stars as well as two X-ray stars. Later, we devoted several experiments to the most active stars. A recent experiment was also conducted to observe southern hemisphere RS CVn in order to increase the astrometric coverage of the celestial sphere. From all these experiments, we detected 9 RS CVn (UX Ari, HR1099, HR5110, $\sigma$ CrB, AR Lac, II Peg, SZ Psc, HD26337, HD77137), as well as Algol and the two X-ray stars LSI $61^{\circ} 303$ and Cyg X1.

Palagi et al (these proceedings) have also conducted Mark III VLBI observations of $4 \mathrm{RS} \mathrm{CVn}$ and Algol at $5 \mathrm{GHz}$ with a multi-station VLB array in 1986.

One of the difficulties in observing RS CVn is that their flux densities are extremely variable on a time scale as short as a day. None of these stars is dependable at a flux density level of $10 \mathrm{mJy}$. However when an outburst occurs, it might last for several days. Depending on the stage of the outburst, the flux density might change on an hour time scale, limiting the hybrid mapping of these stars to snapshots.

\section{c. RESULTS AND INTERPRETATION: CORE-HALO IN CLOSE BINARIES}

Table 1 summarizes the most precise VLBI measurements made for $5 \mathrm{RS}$ CVn and Algol.

\begin{tabular}{|c|c|c|c|c|c|c|}
\hline Star & $\begin{array}{c}\text { Flux } \\
\text { density } \\
(\mathrm{mJy})\end{array}$ & $\begin{array}{l}\text { Measured } \\
\text { angular } \\
\text { size } \\
\text { (mas) }\end{array}$ & $\begin{array}{c}\text { Linear } \\
\text { size } \\
10^{11} \\
\mathrm{~cm}\end{array}$ & $\begin{array}{c}\text { Measured } \\
\text { Brightness } \\
\text { Temp. } \\
\left(10^{8} \mathrm{~K}\right)\end{array}$ & $\begin{array}{l}\text { Magnetic } \\
\text { field } \\
\text { (Gauss) }\end{array}$ & Refer. \\
\hline HR5110 & $15-165$ & $0.9-1.4$ & $4-11$ & $4-20$ & $5-150$ & $a, d$ \\
\hline UX Ari & $14-145$ & $0.4-3.2$ & $3-26$ & $1-100$ & $5-20$ & $b, d$ \\
\hline HR1099 & $12-400$ & $0.3-2.1$ & $4-12$ & $1-300$ & $5-200$ & $b, c, d$ \\
\hline Algol & $5-220$ & $0.5-2.2$ & $2-8$ & $3-300$ & $4-200$ & $\mathrm{~d}, \mathrm{e}$ \\
\hline$\sigma \mathrm{CrB}$ & 14 & 0.6 & 2 & 30 & $5-90$ & d \\
\hline SZ Psc & 37 & 0.6 & 8 & 80 & $5-20$ & $\mathrm{~d}$ \\
\hline
\end{tabular}

Table 1: Source sizes and brightness temperatures directly measured by VLBI for 5 RS CVn and Algol. References: a: Lestrade et al 1984a, b: Mutel et al 1984, c: Lestrade et al 1984b, d: Mutel et al 1985, e: Lestrade et al 1987.

The main result is the discovery that radio emission from these binaries exhibits vari- 
able core-halo structure. The brightness temperature of the core is typically $(1-3) \times 10^{10} \mathrm{~K}$ and the corresponding effective energy of the radiating electrons is $E_{\text {eff }} \sim 5 \mathrm{MeV}$ while the brightness temperature of the halo is typically $(5-10) \times 10^{8} \mathrm{~K}$ with $E_{\text {eff }} \sim 0.5 \mathrm{MeV}$ for the radiating electrons. For the most precise measurements made, the halo component is comparable to the overall size of the binary system while the core is smaller than the K-subgiant which is presumably the active star. These high measured brightness temperatures are incompatible with a thermal process and gyrosynchrotron emission must be invoked, i.e. synchrotron emission from mildly relativistic electrons (Lorentz factor $\leq 10$ ) with a power-law energy distribution.

Figures 2 and 3 are typical visibility curves measured for the two RS CVn systems HR1099 and UX Ari observed during outbursts at $10.6 \mathrm{GHz}$ and $5 \mathrm{GHz}$, respectively (see also Palagi et al in these proceedings for UX Ari). These curves show that the halo is strongly resolved over baselines longer than $\sim 60 \times 10^{6} \lambda$ corresponding to source sizes of 2 - 3 milliarcseconds if the first part of the curve is fitted with a Gaussian brightness distribution. Then, there is a plateau which extends up to baselines as long as $220 \times 10^{6} \lambda$ in Figure 3. This is evidence for a radio core smaller than 0.3 milliarcsecond in HR1099 or smaller than $25 \%$ the diameter of its K-subgiant during these observations (Lestrade et al in preparation).

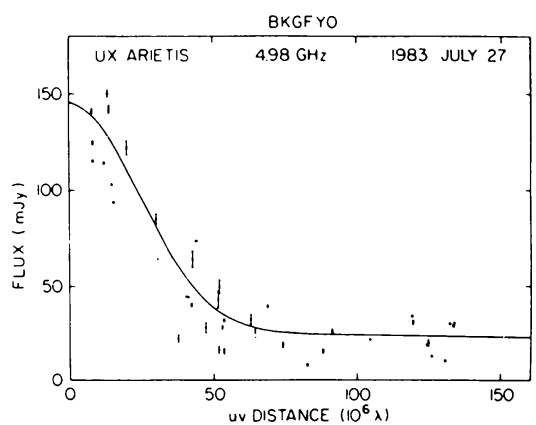

Fig. 2: VLBI visibility curve of UX Ari. Two Gaussian components are fitted to the data (source sizes: 3.2 and $\leq 0.4$ mas)

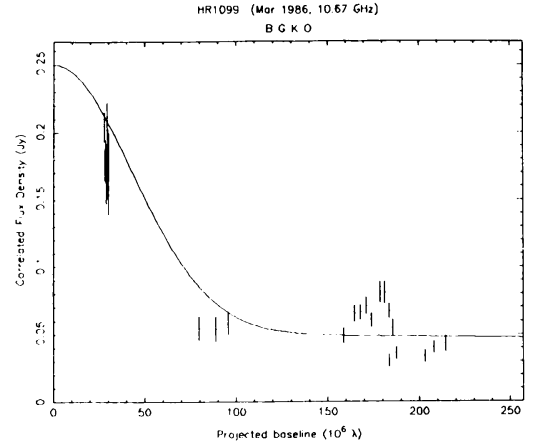

Fig. 3: VLBI visibility curve of HR1099. Two Gaussian components are fitted to the data (source sizes: 1.8 and $\leq 0.3$ mas)

It is interesting to go back to the puzzling result of the experiment designed by Owens and Spangler (1977) to probe the radio source size of the eclipsing RS CVn system AR Lac. Based on the non-thermal and rapidly variable total flux densities of RS CVn, they expected the radio source size to be small. However, they failed to detect any simultaneous optical and radio eclipses and concluded, on the contrary, that the radio source was at least as large as the whole binary system. A compact active core and a large halo found by VLBI reconcile these two seemingly opposite views.

If the gyrosynchrotron process is responsible for the radio emission of $\mathrm{RS} \mathrm{CVn}$ and Algol and if their brightness temperatures $T_{B}$ are measured by VLBI, the magnetic field strength $B$ of the emitting region can be inferred since $T_{\text {eff }} \propto(\nu / 2.8 B)^{0.5+0.5 \delta}$ for this process. In this formula, $\nu$ is the observing frequency, $\delta$ is the energy spectral index of the electron energy distribution $\left(N(E) \propto N E^{-\delta}\right)$ and is assumed to be between 2 and 7 , the effective temperature $T_{\text {eff }}$ is approximately the measured brightness temperature $T_{B}$ if the optical depth of the source is assumed to be close to unity. The ranges of magnetic 
field strengths in Table 1 have been inferred with these assumptions. It is interesting to note that VLBI observations can provide indirect measurements of the magnetic field strengths in stars while this is not possible by any other technique for fields weaker than 1000 Gauss.

A simple physical model accounting for the observed angular size, polarization and spectrum is proposed in Mutel et al 1985 by analogy with some types of "Type IV" radio emission from the Sun. Some energy deposition mechanism (presently unknown) produces enough electrons with energy $>1 \mathrm{MeV}$ in the magnetic structure of the active star to form a small self absorbed (optically thick) gyrosynchrotron source. This core is so small according to our measurements (e.g. < 0.3 milliarcsecond for HR1099) that the energy deposition can occur in a single magnetic loop of the active K-subgiant. As the outburst evolves, this coronal loop expands to a size comparable with the binary system itself. Alternatively, the high energy electrons suffer rapid radiation loss in the active core and are eventually injected with low energy $\left(E_{\text {eff }}<1 \mathrm{MeV}\right)$ in a joint magnetosphere formed of loops connecting between the two stellar components as proposed by Uchida and Sakurai (1983). These low energy electrons can produce an optically thin and circularly polarized halo if the magnetospheric field is sufficiently strong. Such circularly polarized halo associated with an unpolarized core have actually been observed for Algol at $5 \mathrm{GHz}$ (Mutel et al 1985). The radiative lifetime of the high energy electrons in the core is only a few hours while it is several days for the low energy electrons in the halo if the values for $E_{\text {eff }}$ and the magnetic field strengths inferred above are used.

This model agrees with the most interesting data we obtained in July 83 with a six-station VLB array on UX Ari undergoing a strong outburst of $145 \mathrm{mJy}$ at 5 $\mathrm{GHz}$. All the 15 baselines detected the star and the resulting visibilities (including closure phases) were used to map the source with the hybrid mapping technique. This map in Fig. 4 shows a core-halo morphology with the brightness temperature twelve times higher in the core than in the halo. The map shows also that the centroids of the core and halo have a position offset about equal to the projected major axis of the binary at the time of observation. This finding led us to locate the optical components of UX Ari relatively to the radio map as shown in Figure 4 but this is merely a conjecture. The elongated radio halo of Fig. 4 favors the joint magnetosphere model hypothesized by Uchida and Sakurai (1983). The halo contains $80 \%$ of the total flux density and has a size of 3.2 milliarcseconds (almost twice as large as the overall binary size), making it the most extended radio source from RS CVn systems detected so far. The core was unresolved on the longest baseline, indicating a size $<0.4$ milliarcsecond, i.e. smaller than $75 \%$ of the diameter of the

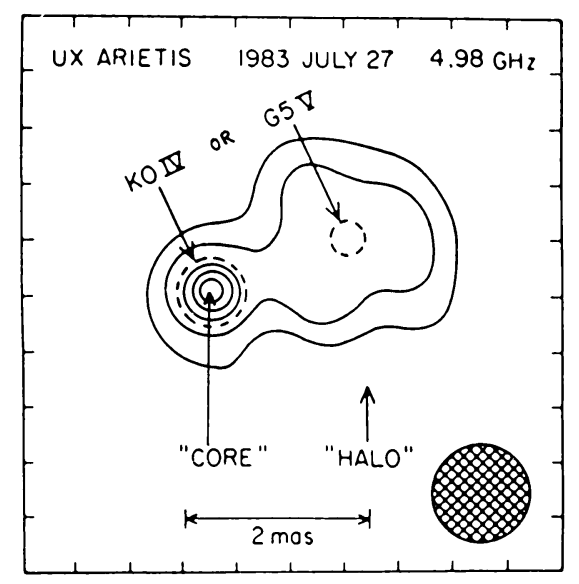

Fig. 4: hybrid map of UX Ari at $5 \mathrm{GHz}$. The contour levels are at $25,35,50,70$ and 90 per cent of the peak brightness. The location of the two optical stellar components relative to the radio map is conjectural (see text). 
K-subgiant.

\section{d. DUAL FREQUENCY AND DUAL POLARIZATION VLBI OBSERVATIONS OF ALGOL}

An exceptional event occurs on Algol when we were conducting VLBI observations simultaneously at 2.3 and $8.4 \mathrm{GHz}$ on 1983 May. The total flux density of Algol changes from 44 to $122 \mathrm{mJy}$ at $2.3 \mathrm{GHz}$ and from 42 to $240 \mathrm{mJy}$ at $8.4 \mathrm{GHz}$ in a 3-hour period. From the pre-outburst to outburst phase, the source size stays constant at $2.3 \mathrm{GHz}(1.9$ mas or $3 R_{K \text {-subgiant }}$ ) while it decreases from 0.6 to 0.45 mas at $8.4 \mathrm{GHz}$. An enhanced total flux density could be due to the expansion of an optically thick radio source in Algol but this is clearly not what we observed in this early stage of the outburst. On the contrary, the source size has decreased at $8.4 \mathrm{GHz}$ implying that it is the brightness temperature

which has increased. This is a direct evidence for energy deposition and production of high energy electrons $(\sim 5 \mathrm{MeV})$ in a region as small as a single loop.

Klein and Chiuderi-Drago (1987) have simulated gyrosynchrotron emission from single loop and loops connecting the components of a typical RS CVn system and found the same frequency dependence of the source size as we observed.

Another exceptional event occurs on Algol when we were conducting VLBI/VLA observations at $1.6 \mathrm{GHz}$ recording simultaneously both senses of circular polarization. Figure 5 shows the total flux density history from the VLA data during 3 scans spaning 6 hours. The first and third scans are only slightly circularly polarized while a highly left circularly polarized outburst $\left(\pi_{c} \sim 50 \%\right)$ occurs during the second scan with a high brightness temperature $\left(>5 \times 10^{9} \mathrm{~K}\right)$ directly measured by VLBI. The combination of large fractional circular polarization $\pi_{c} \geq 50 \%$ and high brightness temperature $\left(>10^{9} \mathrm{~K}\right)$ is difficult to produce by any incoherent gyrosynchrotron mechanism because high $T_{\text {eff }}$ implies both high $E_{\text {eff }}$ of the radiating electrons and low magnetic field while high degree of circular polarization requires the opposite conditions. Melrose and Dulk (1982) propose that such events, which are known on the Sun too, be coherent emission. They developed the theory of an electron cyclotron maser source with radiation escaping at the second harmonic and driven by a loss cone in the foot of a magnetic loop. A magnetic field of 300 Gauss in the maser region is inferred during our observations of Algol from this model.
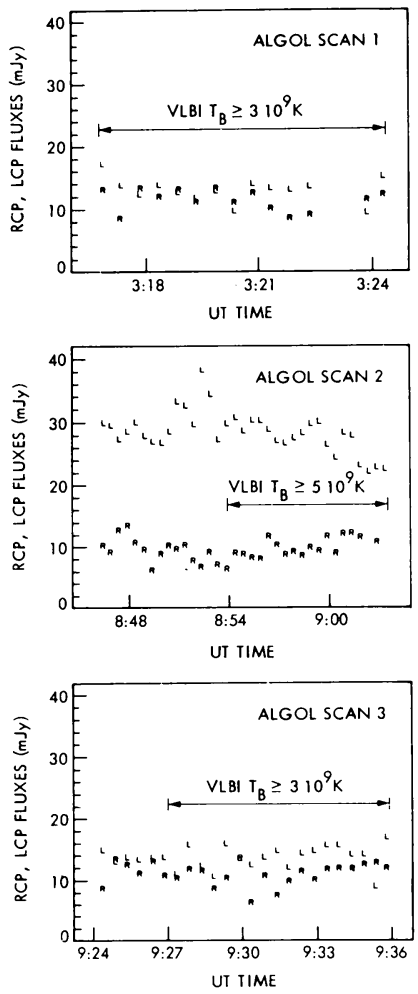

Fig. 5: high brightness temperature associated with a highly circularly polarized outburst of Algol at $1.6 \mathrm{GHz}$. A coherent emission process might be responsible for this event. 


\section{X-RAY BINARIES AND THE EXOTIC OBJECT SS433}

Eleven major known radio emitting X-ray stars are listed in Hjellming and Johnston (1985). These objects are binary systems with X-ray emission from accretion discs. They exhibit relativistic expanding synchrotron sources which are common in extragalactic objects. The periodicities of the X-ray and radio emissions in three of these objects coincide with their orbital periods (LSI $61^{\circ} 303$ (26.5 days), Cir X1 (16.59 days), Cyg X3 (4.95 hours). The formation and evolution of jets in these stellar objects can be studied on a time scale comparable to their orbital periods, i.e. considerably shorter than in extragalactic sources.

Cyg X3: Molnar et al (1984) studied the low-level radio emission which is characterized as a succession of synchrotron flares. The timing of these flares suggests that they are triggered by small variation in the binary separation. Molnar (1986) (see also Molnar in these proceedings) conducted Mark III VLBI observations during one flare of Cyg X3 at $1.3 \mathrm{~cm}$. The VLBI data are consistent with a model composed of an elongated synchrotron source expanding with time at a rate of $0.41 \mathrm{mas} / \mathrm{h}$. Molnar (1986) proposes that the expansion rate corresponds to the tranverse velocity of a double-sided jet.

Cyg X1: this black hole candidate binary was detected at $5 \mathrm{GHz}$ on 1983 July 26 . The $15 \mathrm{mJy}$ source was unresolved and the upper limit of its source size is 0.5 milliarcsecond (Mutel et al 1985). The data were not sufficient to probe the morphology of the source.

LSI $61^{\circ} 303$ : this binary (Be star + neutron star ?) is a periodic non-thermal radio source. It has been detected on VLBI baselines at $1.6 \mathrm{GHz}$ on 1983 October 16 during its quiescent period ( $25 \mathrm{mJy}$ ). A Gaussian brightness distribution was fitted to the VLBI visibility function yielding a source size of 4.1 mas (unpublished). At 2300 parsecs, this size is twelve times the major axis of the binary system. It is not clear if such a large source size is due to interstellar scattering or if radiating electrons in the environment of the binary are accelarated by Compton collision with $\gamma$-rays produced by the neutron star (Vestrand 1983).

Circ X1: this periodic radio emitting binary was observed on VLBI baselines at $2.3 \mathrm{GHz}$ by Preston et al (1983) to measure its source size during the quiescent period (size $>0 " .2$ ) and during flaring (size between $0 " .0015$ and $0 " .015$ ). As for LSI $61^{\circ} 303$, it is not clear if interstellar scattering or relativistic expansion of a jet is responsible for such a large source.

Sco X1: Geldzahler and Fomalont (these proceedings). report on the first epoch Mark III VLBI observations of the variable radio source Sco X1 to determine the proper motions of the core and of the two surrounding lobes.

SS433: the optical emission lines of H I and He I in the SS433 star system (V1343 Aql) have twin Doppler shifts, corresponding to velocity of $80000 \mathrm{~km} / \mathrm{s}$, with a period of 164 days. A precessing twin-jet model was proposed for these optical data but the two angles between the ejection axis and precession axis and between the precession axis and the line of sight cannot be unambiguously determined from optical data. Walker et al (1981) conducted VLBI observations of SS433 and found that the data were consistent with a model composed of a compact core and of collinear elongated jets of $\sim 0$ ".1 and 2 " in length. They found that the extended components are approximately aligned with the outer bulges of the super nova remnant W50 on which SS433 is superposed, suggesting a physical association between SS433 and the distorsion of the super nova remnant. Niell et al (1981) conducted extensive VLBI observations at $2.3 \mathrm{GHz}$ at 12 epochs over 15 months. They found that the variation of the position angle of the elongated components has a sinusoidal signature corresponding to the optical period and that the position angle of this radio component lags that of the optical model by 17 days. These observations provide physical evidence for precessing jets, a model which has been suggested to explain the misalignment of the small and large scale structures of extragalactic sources. These authors 
also resolved the ambiguities in the two angles of the optical model of SS433 (see also the VLBI observations of Schilizzi et al 1981). From a subset of their VLBI observations, Niell et al (1981) found evidence for motion of individual radiating knots and combined the proper motion of these blobs with the absolute ejection velocity $(0.26 \mathrm{c})$ obtained from the optical data to determine the distance of SS433 $(5.1 \mathrm{kpc})$. Vermeulen et al (these proceedings) have conducted six Mark III VLBI experiments at two-day intervals to study the properties of the individual knots and of the ambient medium in which they move.

\section{ASTROMETRY WITH RADIO STARS}

The primary celestial reference frame is now based on precise VLBI positions of extragalactic sources. This is the most stable system because quasars or galactic nuclei have no detectable proper motions. However, this system is not dense $(\sim 200$ sources for the whole sky) and is not as accessible as the optical system which is based on the positions of several thousands stars. Neverthless, the optical system lacks stability and so it is beneficial to tie the two frames together. For example, this process directly removes the global rotation of the optical system due to the galactic rotation. The global angular offsets and rates can be determined by comparing the radio positions (unit-vector: $\sigma_{v l b i}$ ) and radio proper motions $\left(\dot{\sigma}_{v l b i}\right)$ referred to the VLBI reference frame with the corresponding optical quantities $\left(\sigma_{\text {opt }}\right.$ and $\left.\dot{\sigma}_{\text {opt }}\right)$ of radio stars. The RS CVn binaries and some X-ray stars are optically bright (magnitude $<11$ ) and sufficiently active in radio to be used as link stars. The following relations hold for each link star:

$$
\boldsymbol{\sigma}_{v l b i}=[R] \boldsymbol{\sigma}_{\text {opt }} \quad ; \quad \dot{\sigma}_{v l b i}=[R] \dot{\boldsymbol{\sigma}}_{\text {opt }}+[\dot{R}] \boldsymbol{\sigma}_{\text {opt }}
$$

The matrices $[R]$ and $[\dot{R}]$ are functions of the 3 angular offsets and rates. These parameters can be solved for with the above set of equations if enough positions of radio stars are measured.

Niell et al (these proceedings) have determined VLBI positions of 8 radio stars with astrometric uncertainties ranging from 2 to 40 milliarcseconds. These positions are derived from VLBI delays and fringe rates and their astrometric precision strongly depends on their flux densities. Lestrade et al (these proceedings) have used a phase reference VLBI technique to determine the relative position between a reference source and Algol in a very low state of activity $(4 \mathrm{mJy})$ by coherently integrating 4.1 hours of VLBI data. The uncertainty on their relative position is estimated to be about twice the stochastic precision of 0.8 milliarcsecond (anzular resolution / SNR). This technique makes at least a dozen radio stars always available for differential astrometry.

Linking the optical and radio celestial reference frames is crucial for the Hipparcos space mission which should provide a new optical celestial frame as precise as the VLBI system (i.e. milliarcsecond precision) in the 90 's.

\section{PROSPECTS}

Is radio emission from $\mathrm{RS} C \mathrm{CV}$ a local phenomena, occuring in a single coronal loop of the subgiant for instance, or is it a global phenomena, involving the two stellar components through connecting magnetic loops for instance?. Klein and Chiuderi-Drago (1987) have simulated both models and concluded that a structure interconnecting regions of intense magnetic fields on both stars of the binary should be revealed by a double structure of the radio source (two hot spots) at high frequency $(>10 \mathrm{GHz}$ ). These two possible models could be discriminated by high dynamics range VLBI maps. Another issue is how large is the active core where outbursts are triggered by some energy deposition mechanism?. 
The cores observed so far are unresolved on terrestrial baselines $>100 \times 10^{6} \lambda$ for UX Ari and Algol and even baselines $>200 \times 10^{6} \lambda$ for HR1099. High frequency or orbiting VLBI observations might provide new insights.

Ultra-precise differential astrometry $(<100 \mu a s)$ repeated over hour, day, month and year intervals should separate between intrinsic motion of the active core, orbital motion, space motion of the whole system (proper motion) and trigonometric parallax. If the core is stable in a loop attached to the subgiant, its motion around the gravity center of the binary system could be used to measure the major axis by repeated VLBI astrometric measurements. Then, individual masses of the components could be determined. Mass is one of the most difficult parameter to measure in stellar physics.

VLBI investigations should be initiated on new classes of stars which are susceptible to exhibit milliarcsecond structures, e.g. dMe, FK Com and T Tauri stars.

\section{Acknowledgements}

The research described in this report was carried out, in part by the Jet Propulsion Laboratory of the California Institute of Technology, under contract with the National Aeronautics and Space Administration.

References

Abbott, D. C., 1985, in "Radio Stars", Workshop held in Boulder August 1984, edited by R. M. Hjellming and D.M. Gibson, 61.

Clark, B.G., Kellermann, K.I., Shaffer, D.B., 1975, Ap. J., 198, L123.

Clark, T.A. et al, 1976, Ap. J.(Letters), 206, L107.

Dulk, G.A., 1985, An. Rev. Astron. and Astroph, vol 23, 169.

Hall, D.S., 1975, in "Multiple Periodic Variable Stars", IAU Colloquium No 29, Budapest.

Hjellming, R.M., Johnston, K.J., 1985, in "Radio Stars", Workshop held in Boulder August 1984, edited by R. M. Hjellming and D.M. Gibson, 61 .

Klein, K.L., Chiuderi-Drago, F., 1987, Astron. Astrophys. (in press)

Kwok, S., 1985, in "Radio Stars", Workshop held in Boulder August 1984, edited by R. M. Hjellming and D.M. Gibson, 79.

Lestrade, J.-F., Mutel, R.L., Preston, R.A., Scheid, J.A., Phillips, R.B., 1984a, Ap. J., $279,184$.

Lestrade, J.-F., Mutel, R.L., Phillips, R.B., Niell, A.E., Preston, R.A., 1984b,Ap. J. (Letters), 282, L23.

Lestrade, J.-F., Mutel, R.L., Preston R.A., Phillips, R.B., 1987, submitted Ap.J.

Little-Marenin, I.R., Simon, T., Ayres, T.R., Cohen, N.L., Feldman, P.A., Linsky, J.L., Little, S.J., Lyons, R., 1986, Ap. J., 303, 780 .

Ma, C., et al, 1986, A.J., 92, 1020.

Melrose, D.B., Dulk, G.A., 1982, Ap. J.,259, 844.

Molnar, L.A., Reid, M.J., Grindlay, J.E., 1984, Nature, 310, 662.

Molnar, L.A., 1986, in "Physics of Accretation onto Compacts Objects", held in Tenerife (Spain) April 1986, M. Watson K. Mason and N. White (eds)

Mutel, R.L., Doiron, D., Lestrade, J.-F., Phillips, R.B., 1984, Ap. J., 278, 220.

Mutel, R.L., Lestrade, J.-F., Preston, R.A., Phillips, R.B., 1985, Ap. J., 289, 262.

Mutel, R.L., Lestrade, J.-F. , 1985, , A.J., 90, 493.

Mutel, R.L., Morris, D.H., Doiron D.J., Lestrade, J.-F., 1987, A. J., 93, , 1220.

Niell A.E., Lockhart, T.G., Preston, R.A., 1981, Ap. J., 250, 248.

Owens, F.N., Spangler, S.R., 1977, Ap. J., 217, L41.

Pallavicini, R., 1986, Highlights of Astronomy IAU 1986, edited by J.P. Swings, 447.

Preston, R.A. et al, Ap. J., 268, L23.

Rodonò, M., 1986, Highlights of Astronomy IAU 1986, edited by J.P. Swings, 429.

Rogers, A.E.E. et al, 1983, Science, $219,51$.

Uchida, Y., Sakurai, T., 1983, in "Activity in Red Dwarf Stars, IAU Colloq. 71, edited by M. Rodonò, Reidel, 629.

Schilizzi, R. T., Miley, G.K., Romney, J. D., Spencer, R.E., 1981, Nature, 290, 318.

Vestrand, W. T., 1983, Ap. J., $271,304$.

Vogt, S.S., Penrod, G.D., 1983, P.A.S.P., 95, 565.

Walker, R.C., et al, 1981, Ap. J., 243, 589.

Walter F.M., Gibson, D.M., Basri, G.S., 1983, Ap. J., 267, 665

White, N.E. et al, 1985, in "Cool Stars, Stellar Systems and the Sun", edited by D. Gibson and M. Zeilik. 